\begin{tabular}{ll|l}
\cline { 2 - 3 } & \multicolumn{2}{l}{ Intervent Neurol 2017;6:16-24 } \\
\hline $\begin{array}{l}\text { DOI: 10.1159/000449321 } \\
\text { Published online: October 7, 2016 }\end{array}$ & $\begin{array}{l}\text { (C) 2016 S. Karger AG, Basel } \\
\text { www.karger.com/ine }\end{array}$ \\
\hline
\end{tabular}

\title{
Stent Retriever-Mediated Manual Aspiration Thrombectomy for Acute Ischemic Stroke
}

\author{
Ashutosh P. Jadhav ${ }^{a, b} \quad$ Amin Aghaebrahim $^{a} \quad$ Anat Horev $^{d}$ \\ Dan-Victor Giurgiutiuc ${ }^{c}$ Andrew F. Ducruet ${ }^{b}$ Brian Jankowitz ${ }^{b}$ \\ Tudor G. Jovin ${ }^{a}$ b \\ Departments of a Neurology and beurological Surgery, UPMC Stroke Institute, University \\ of Pittsburgh Medical Center, Pittsburgh, Pa. and 'Winchester Neurological Consultants, \\ Winchester, Va., USA; d Soroka Medical Center, Beersheba, Israel
}

\section{Key Words}

Acute stroke $\cdot$ Stent retriever $\cdot$ Manual aspiration $\cdot$ Intervention $\cdot$ Revascularization

\begin{abstract}
Background and Purpose: Stent retriever thrombectomy and manual aspiration thrombectomy (MAT) have each been shown to lead to high rates of recanalization as single-modality endovascular stroke therapy. We sought to describe the safety and efficacy of a multimodal approach combining these two techniques termed 'stent retriever-mediated manual aspiration thrombectomy' (SMAT) and compared them to MAT alone. Methods: Retrospective review of a prospectively acquired acute endovascular stroke database. Results: 195 consecutive patients with large-vessel occlusion were identified between July 2013 and April 2015. Occlusion distribution was as follows: 52\% middle cerebral artery segment 1 (M1), 6\% M2, 29\% internal carotid artery, and $13 \%$ vertebrobasilar. Median onset to treatment time was $278 \mathrm{~min}$. Intravenous rtPA was administered in 33\% of cases, whereas $34 \%$ of cases had symptom onset beyond $8 \mathrm{~h}$. Effective recanalization (TICI 2b/3) was achieved in $91 \%$ of patients and in $49 \%$ of patients, only a single pass was necessary. Median groin puncture to recanalization time was $40 \mathrm{~min}$. Symptomatic intracerebral hemorrhage occurred in $5 \%$ of patients. Favorable outcomes defined as a modified Rankin Scale score of $0-2$ were noted in $42 \%$ of patients. Compared with MAT alone, SMAT achieved a similar rate of effective recanalization (91 vs. $88 \%$, p = n.s.) but was associated with faster access to reperfusion times (49 vs. $77 \mathrm{~min}, \mathrm{p}<0.00001$ ). Conclusions: SMAT is a safe and efficacious method to achieve rapid revascularization that leads to faster recanalization compared to manual aspiration alone. Future prospective comparisons are necessary to establish the most clinically effective therapy for acute thrombectomy.
\end{abstract}




\section{Introduction}

In patients presenting with acute ischemic stroke secondary to large-vessel occlusion, endovascular therapy leads to superior outcomes compared to medical therapy. Recent trials have demonstrated that in appropriately selected patients treated with efficient workflow (fast door to imaging and imaging to groin puncture time) and modern thrombectomy devices yielding high rates of effective (thrombolysis in cerebral infarction [1], TICI 2b/3) recanalization, rapid reperfusion is of critical importance for obtaining superior outcomes. Of the 5 recently published endovascular trials [2,3], the use of stent retrievers was required in 3 of the trials and heavily represented in the other 2 trials with TICI $2 b / 3$ rates in the range of $59-88 \%$. This represents a significant improvement compared to the TICI $2 \mathrm{~b} / 3$ rate of $42.5 \%$ observed in the Interventional Management of Stroke III Trial (IMS III) [4]. While superior to previous mechanical or pharmacological approaches, prospective case series revealed that with effective reperfusion rates in the $68-69 \%$ range, the need for multiple passes to achieve recanalization and the incidence of embolization in previously uninvolved territory (ENT), stent retriever embolectomy can be further improved. Aspiration thrombectomy performed either manually [5, 6] or in conjunction with a pump [7] has been described as a therapeutic option with signals of benefit over intravenous rtPA as a stand-alone treatment in a recently conducted randomized trial [8], although the therapeutic effect seems lower with this technology compared with stent retrievers. We have previously reported on the role of manual aspiration thrombectomy (MAT) as an adjunctive therapy [5] or as a primary first-pass technique [6] in large-vessel occlusion. Despite a high rate of effective reperfusion observed with primary MAT, in light of the high rates and quality of recanalization observed with stent retriever technology, we modified our manual aspiration technique to include an initial step of stent retriever deployment followed by aspiration. Here, we report our experience with consecutive cases using a combined modality approach of stent retriever-mediated manual aspiration thrombectomy (SMAT) and compare its efficacy to historical controls treated with first-line MAT therapy.

\section{Methods}

\section{Patient Selection}

This study was approved by the University of Pittsburgh Institutional Review Board. A retrospective review of a prospectively maintained database of acute ischemic stroke was performed to identify patients who underwent intra-arterial therapy with SMAT. Patients were selected for thrombectomy by a vascular neurologist based on significant or disabling clinical deficit, evidence of small core infarct [CT ASPECT score (Alberta Stroke Program Early CT score) >5 [9], CT perfusion-measured core volume by RAPID or MRI DWI $<50 \mathrm{~cm}^{3}$ ] and presence of large-vessel occlusion [middle cerebral artery segment 2 (M2), M1, internal carotid artery (ICA) or basilar occlusion] on CT angiography, MR angiography or CT head (hyperdense sign). Advanced age or long time of symptom onset alone were no exclusion criteria; however, all patients had to have premorbid independence (modified Rankin Scale score of 0-2).

\section{Procedural Technique}

Access was achieved with a transfemoral approach in a majority of patients. A 6-Fr base catheter (Neuron Max, Penumbra, Alameda, Calif., USA) was advanced to the descending aortic arch under road map guidance. A long diagnostic catheter and guide wire were then advanced into the target common carotid artery or vertebral artery and the base catheter was serially advanced to the cervical carotid or distal V2 segment. A coaxial system (fig. 1a) consisting of a mid-sized catheter (072 Navien or 058 Navien, Covidien Vascular Therapies, or 5 Max ACE, Penumbra, Alameda, Calif., USA) and microcatheter (Marksman, Velocity, Penumbra, Alameda, Calif., USA, or 18L, Stryker, Kalamazoo, Mich., USA) with an 0.014-in Synchro 2 or 0.016-in Fathom microwire (Covidien Vascular Therapies) is then introduced into the base catheter and advanced up to the cavernous segment under road map guidance. The base catheter is then advanced as 
Jadhav et al.: Stent Retriever-Mediated Manual Aspiration Thrombectomy for Acute Ischemic Stroke

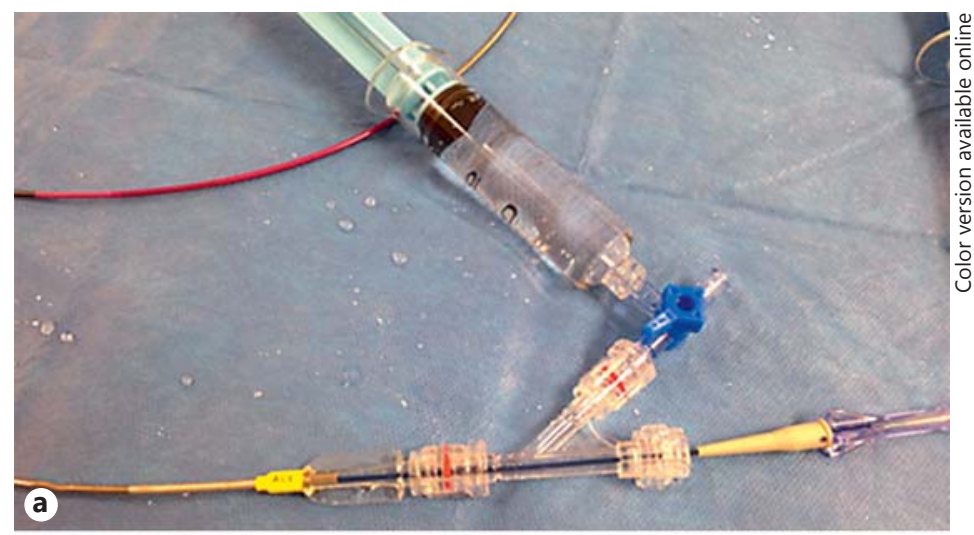

Fig. 1. A coaxial system consisting of a mid-sized distal access catheter attached to a 3-way valve and microcatheter (a) is advanced to access the intracranial occlusion. The microwire is shaped in a ' $\mathrm{J}$ ' shape to minimize likelihood of wire perforation (b). A clot is retrieved from the stent retriever device as well as the aspiration catheter tip (c).

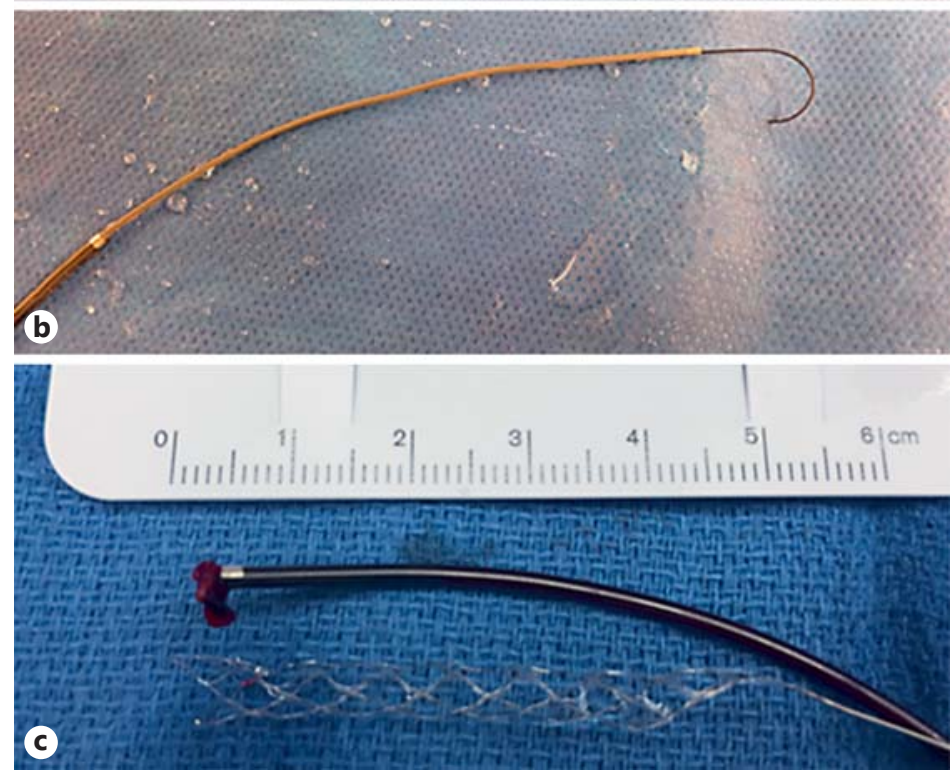

distal as possible, typically up to the petrous or proximal cavernous carotid or proximal V3 segment. The target lesion is crossed with the microwire/microcatheter and the microwire (fig. 1b) is carefully withdrawn. A microcatheter injection was performed to confirm the appropriate intravascular position of the microcatheter distal to the clot and the stent retriever device was advanced up to the microcatheter tip. The stent retriever device (Solitaire, Covidien Vascular Therapies; Trevo, Stryker) was then slowed unsheathed while withdrawing the microcatheter. During this time, the mid-sized catheter is maintained proximal to the occlusion, generally in the cavernous carotid. The stent retriever device was deployed for 5 min after which the mid-sized catheter is advanced towards the clot, which is facilitated by traction on the deployed stent retriever. Once the mid-size catheter reaches the proximal aspect of the clot, the stent retriever is withdrawn out of the body together with the microcatheter while applying suction to a $20-\mathrm{ml}$ syringe. Subsequently, the aspiration catheter is slowly withdrawn under continuously applied aspiration and assessed for thrombus (fig. 1c). Additional aspiration was performed from the base catheter prior to an angiographic run.

\section{Patient, Radiographic and Procedural Characteristics}

Baseline patient characteristics included age, gender, clinical deficit [National Institutes of Health Stroke Scale (NIHSS)] and medical history (presence or absence of diabetes, hypertension, hyperlipidemia, tobacco use, atrial fibrillation, coronary artery disease). Radiographic characteristics included CT ASPECT score, site of vessel occlusion (M2, M1, ICA terminus, tandem ICA-MCA and basilar artery). Procedural workflow was measured at time intervals of groin puncture to base catheter placement and base catheter placement to first successful reperfusion (TICI $2 \mathrm{~b}$ or better). 
Table 1. Baseline characteristics $(\mathrm{n}=195)$

\section{DOI: $10.1159 / 000449321$}

Jadhav et al.: Stent Retriever-Mediated Manual Aspiration Thrombectomy for Acute Ischemic Stroke

\begin{tabular}{ll}
\hline Women, \% (n) & $48(95)$ \\
Age, years & $69 \pm 15$ \\
Hypertension, \% (n) & $73(142)$ \\
Diabetes mellitus, \% (n) & $16(31)$ \\
Dyslipidemia, \% (n) & $45(88)$ \\
Tobacco use, \% (n) & $25(49)$ \\
Atrial fibrillation, \% (n) & $34(66)$ \\
Coronary artery disease, \% (n) & $29(57)$ \\
Admission NIHSS score & $17 \pm 5$ \\
Presenting within 8 h of symptoms onset, \% & 65 \\
Site of large-vessel occlusion, \% & \\
$\quad$ Posterior circulation & 13 \\
$\quad$ ICA tandem & 11 \\
$\quad$ ICA terminus & 18 \\
M1 & 52 \\
M2 & 6 \\
Left-sided lesion, \% & 47 \\
Median CT ASPECT score & 9 \\
CT ASPECT score 9-10, \% & 54 \\
Qualifying image, \% & \\
MRI & 25 \\
CT angiography & 76 \\
CT perfusion & 59 \\
\end{tabular}

Statistical Analysis

Statistical analysis was performed using the STATA IC 10 software (StataCorp LP, College Station, Tex., USA). Descriptive statistics were obtained. In univariate analysis, several variables of interest were correlated to good functional outcome. For each end point, all covariates with a probability value $<0.2$ were then entered into a multivariate stepwise logistic regression model. Significant association was considered for a probability value of $<0.05$. Additional comparisons were made to a prior series of pure MAT [5] by the $\chi^{2}$ test.

\section{Results}

\section{Patient Characteristics}

Baseline characteristics are reported in table 1. Advanced age was not a contradiction to intra-arterial therapy, with $24 \%$ of patients being 80 years of age or older. A third of the patients were treated beyond $8 \mathrm{~h}$. Reflective of the referral patterns at our institution, 59\% of patients were transferred from outside facilities. Patient selection was based on a small core observed on a preprocedural scan in the presence of a significant clinical deficit. The majority of patients underwent a combination of CT angiography and perfusion. MRI was used for selection in $25 \%$ of patients and CT head alone without additional contrast studies was utilized in $11 \%$ of patients. A majority of patients with anterior circulation strokes had a favorable CT ASPECT score on presentation (CT ASPECT score of 9 or 10). Anterior circulation strokes accounted for $87 \%$ of the treated patients.

\section{Procedural Details}

The procedures were typically conducted under conscious sedation with $14 \%$ of patients requiring general anesthesia due to medical reasons or due to being intubated prior to arrival at our hospital. Procedural details are reported in table 2 . Alternative access ( 1 transradial, 1 


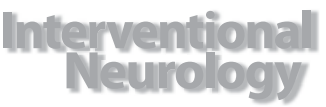

Table 2. Procedural details

\begin{tabular}{l|l}
\hline Intervent Neurol 2017;6:16-24 \\
\hline DOI: 10.1159/000449321 & $\begin{array}{l}\text { @ 2016 S. Karger AG, Basel } \\
\text { www.karger.com/ine }\end{array}$ \\
\hline
\end{tabular}

Jadhav et al.: Stent Retriever-Mediated Manual Aspiration Thrombectomy for Acute Ischemic Stroke

\begin{tabular}{lc}
\hline Alternative access & $2 \%$ \\
Stent & $14 \%$ \\
$\quad$ Intracranial & $46 \%$ \\
Mid-sized catheter & \\
$\quad$ Navien 072 & $53 \%$ \\
$\quad$ Navien 058 & $12 \%$ \\
$\quad$ MAX ACE & $35 \%$ \\
Microcatheter & \\
$\quad$ 18L & $5 \%$ \\
$\quad$ Marksman & $68 \%$ \\
$\quad$ Velocity & $27 \%$ \\
Stent retriever & \\
$\quad$ TREVO & $17 \%$ \\
$\quad$ Solitaire & $83 \%$ \\
Trevo $3 \times 20$ & 2 \\
Trevo $4 \times 20$ & 31 \\
Trevo $6 \times 25$ & 3 \\
Solitaire $4 \times 20$ & 11 \\
Solitaire $4 \times 40$ & 6 \\
Solitaire $6 \times 30$ & 144 \\
\hline
\end{tabular}

transcervical, 1 transaxillary) was necessary in 3 cases due to femoral artery and aortic arch disease. The median time from groin access to base catheter run was $13 \mathrm{~min}$. The largest bore intermediate catheter was selected in a majority of cases with the use of the Navien catheter in $65 \%$ of cases (88\% 072 Navien and $12 \%$ Navien 058) and 5 Max ACE in 35\% of cases. The Solitaire stent retriever was utilized in $87 \%$ of cases and the Trevo device in the remaining cases. Adjunctive stent placement was utilized in $14 \%$ of patients with intrinsic lesions of whom $46 \%$ underwent intracranial stent placement (majority of basilar occlusions with underlying atherosclerotic lesions). Workflow details are outlined in table 3 . In patients with successful recanalization $(n=177)$, base catheter to reperfusion required a median of $24 \mathrm{~min}$ with no significant differences related to the selection of the intermediate catheter or stent retriever. In anterior circulation stroke, mean puncture to reperfusion time was $38 \mathrm{~min}$, while in the posterior circulation stroke it was $73.5 \mathrm{~min}(\mathrm{p}<0.0001)$.

\section{Features of Nonrecanalizers}

Of the 18 patients that could not be successfully recanalized, there was a higher association with ICA occlusions ( 45 vs. $28 \%$ ), late presentation ( $50 \mathrm{vs.} 34 \%$ ) and lower use of intravenous rtPA ( 25 vs. $34 \%$ ), suggesting an overall larger and more organized thrombus burden.

\section{Radiographic and Clinical Outcomes}

Successful recanalization was achieved in $91 \%$ of cases, with $49 \%$ of patients achieving TICI $2 \mathrm{~b} / 3$ recanalization after a single attempt with SMAT. Perforations occurred in $3 \%$ of cases. Additional procedural complications included 2 cases of nonflow limiting dissection, 1 case of carotid-cavernous fistula formation and 1 case of unintentional stent retriever retention. Postprocedural parenchymal hemorrhage (PH1/2) [10] was observed in 5\% of cases. ENT was defined as any new vessel occlusion or infarct in a previously unaffected territory and was noted in $3.6 \%$ of cases.

Outcomes are detailed in table 4. Overall good outcomes were observed in 42\% (mRS score $0-2$ at 90 days). In patients with anterior circulation stroke and a CT ASPECT score of 9 or 10 , the rate of good outcomes was $54 \%$. There were slightly better outcomes in patients presenting within $8 \mathrm{~h}$ or receiving intravenous rtPA and slightly worse outcomes in patients 
Jadhav et al.: Stent Retriever-Mediated Manual Aspiration Thrombectomy for Acute Ischemic Stroke

Table 3. Workflow: puncture to base catheter (P2B), base catheter to recanalization (B2R), puncture to recanalization (P2R)

\begin{tabular}{lrllll}
\hline Group & $\mathrm{n}$ & P2B, min & B2R, min & P2R, min & First pass, \% \\
\hline Total & 177 & 13 & 24 & 40 & 49 \\
Age $<80$ & 135 & 13 & 25 & 41 & 49 \\
Age $\geq 80$ & 42 & 13 & 23 & 35.5 & 48 \\
Posterior circulation & 24 & 30 & 29 & 73.5 & 58 \\
Anterior circulation & 153 & 12 & 24 & 38 & 47 \\
Solitaire & 149 & 13 & 25 & 40 & 50 \\
Trevo & 28 & 13 & 19.5 & 43.5 & 39 \\
Navien & 113 & 13 & 22 & 37 & 49 \\
5 Max ACE & 64 & 13 & 27.5 & 41.5 & 48 \\
\hline
\end{tabular}

Table 4. Outcomes

\begin{tabular}{lrl}
\hline Group & $\mathrm{n}$ & mRS score 0-2, \% \\
\hline Total & 195 & 42 \\
CT ASPECT score 9-10 & 91 & 54 \\
Presenting within 8 h & 54 & 46 \\
Intravenous tPA recipients & 62 & 44 \\
ICA occlusion & 57 & 40 \\
Posterior circulation & 26 & 38 \\
\hline
\end{tabular}

with basilar occlusions. Overall mortality was $26 \%$, with $38 \%$ mortality in patients with posterior circulation occlusions.

In univariate analysis, TICI 2b/3 recanalization (OR 6.2, 95\% CI 2.14-18.3) and lower number of device passes (OR 8.28, 95\% CI 0.71-0.97) were associated with good outcomes. In univariate and multivariate analysis, good outcomes were associated with younger age (OR 0.97, 95\% CI 0.96-0.99), small core (CT ASPECT score 9-10; OR 1.6, 95\% CI 1.27-1.91) and lower NIHSS scores on presentation (OR 0.87, 95\% CI 0.83-0.92).

\section{Comparison of SMAT to MAT}

In order to compare the SMAT technique to MAT alone, we compared the outcomes associated with this SMAT technique to those in a previously reported series of 112 consecutive patients who had undergone MAT alone at our institute [5]. There was no statistical difference in age, gender, intubation status, stroke risk factors and baseline NIHSS scores between the 2 groups (table 5). However, there was a comparable rate of high-quality recanalization (91 vs. $88 \%, p=$ n.s.), but a significantly faster groin puncture to recanalization time with SMAT ( 49 vs. $77 \mathrm{~min}, \mathrm{p}<0.00001$ ). No statistical difference was found in the rate of good outcomes (42 vs. $46 \%, p=$ n.s.).

\section{Discussion}

Rapid and high-quality reperfusion in the setting of cerebral ischemia is a critical predictor of good outcome $[11,12]$. In contrast to the coronary vasculature, the cerebral circulation is more fragile and arborized with higher burden and heterogeneity of clot composition. Historically, this has led to significantly lower rates of recanalization. Between 2005 and 2009, 


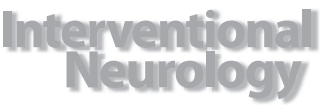

Table 5. SMAT versus MAT

\begin{tabular}{l|l}
\hline Intervent Neurol 2017;6:16-24 \\
\hline DOI: 10.1159/000449321 & $\begin{array}{l}\text { @ 2016 S. Karger AG, Basel } \\
\text { www.karger.com/ine }\end{array}$ \\
\hline
\end{tabular}

Jadhav et al.: Stent Retriever-Mediated Manual Aspiration Thrombectomy for Acute Ischemic Stroke

\begin{tabular}{llll}
\hline Group & MAT & SMAT & p value \\
\hline Sample size & 112 & 195 & \\
Age, years & 66.5 & 69 & n.s. \\
NIHSS score & 17 & 17 & n.s. \\
Intravenous tPA, \% & 41 & 44 & n.s. \\
Passes, n & 2.24 & 2.25 & n.s. \\
Good outcomes, \% & 46 & 42 & n.s. \\
TICI 2b/3, \% & 91 & 88 & n.s. \\
G2R, min & 70 & 49 & $<0.00001$ \\
\hline
\end{tabular}

$\mathrm{G} 2 \mathrm{R}=$ Groin to recanalization

cardiac reperfusion rates were $78.6-86.1 \%$ for complete to near-complete reperfusion compared to $23.4-61.1 \%$ for cerebral reperfusion [13]. Two trials [14, 15] comparing stent retriever devices to the Merci device revealed significantly higher rates of complete or nearcomplete reperfusion (68-69\%) compared to the Merci clot retrieval device (30-44\%). In the STAR registry [16], the use of the Solitaire device led to a $79.2 \%$ rate of TICI $2 \mathrm{~b} / 3$ recanalization rates. The recently completed endovascular clinical trials compared thrombectomy to medical management and exclusively or predominantly utilized stent retriever devices with resultant high rates of recanalization (58-88\%). Overall, stent retrievers represent a significant advancement in high-quality recanalization; however, while cardiac perfusion rates are consistently above $75 \%$, the cerebral reperfusion rates continue to be variable suggesting a need for refinement of the stent retriever approach.

Aspiration thrombectomy has been used as an alternative strategy in acute stroke interventions with adjunctive clot disruption, pump vacuum or manual suction with high rates of recanalization (79-86\%) $[5,6]$. In vitro models have suggested that aspiration alone or adjunctive aspiration may lead to lower rates of emboli in new territories compared to stent retriever thrombectomy. Indeed, prior stent retriever alone studies revealed ENT rates of $7-11 \%[16,17]$. With a combined technique of manual aspiration and stent retriever thrombectomy, we observed very high TICI $2 \mathrm{~b} / 3$ rates of $91 \%$ with a low rate of ENT (3.4\%).

The North American Solitaire Stent Retriever Acute Stroke registry was a prospective postmarketing registry of 354 patients undergoing stent retriever thrombectomy. A subanalysis of the study revealed faster and higher-quality recanalization with ultimately better clinical outcomes in patients treated with balloon guide catheters compared to regular guide catheters [18]. While the reasons are not entirely clear, the proposed mechanism of improved outcomes is felt to be related to proximal flow arrest during device retrieval. The use of a distal aspiration catheter likely abrogates the need for a balloon guide catheter as local aspiration during device retrieval serves to provide proximal protection. Additionally, the use of an intermediate large bore catheter facilitates the delivery of the stent device by providing additional distal support. Furthermore, the stent retriever device serves as an 'anchor' to facilitate distal tracking of the aspiration catheter to the face of the clot [19]. This complementary approach likely accounts for the markedly faster groin to recanalization time.

Our study has several limitations given that the results represent a self-adjudicated, single-center experience, which is retrospective in nature. A previous retrospective smaller, multicenter series of patients undergoing direct aspiration with the stent retriever device similarly showed high rates of recanalization (88\%) and fast recanalization (54 min) [20]. Our study further validates these results. Additionally, we are able to compare the SMAT approach and the MAT approach at a single center with the same pool of operators employing both techniques over two time intervals (MAT, 2012-2013; SMAT, 2013-2015). Despite high 
rates of recanalization and faster treatment times, we found a good outcome rate of $42 \%$. This is within the range of good outcomes observed in the recently published endovascular trials (39.6-71\%). Additionally, our series contains a high percentage of patients with advanced age, late presentation and posterior circulation pathology. Given the advent of larger and more trackable aspiration devices, the efficiency of aspiration will likely continue to increase. A larger study directly comparing stent retriever alone, SMAT and MAT is necessary to determine whether one technique would lead to improved outcomes.

\section{Authors' Contributions}

Authors' contributions to the study and manuscript preparation include the following. Conception and design: all authors. Acquisition of data: all authors. Analysis and interpretation of data: all authors. Drafting the article: Jadhav. Critically revising the article: all authors. Reviewed submitted version of the manuscript: Jadhav. Approved the final version of the manuscript on behalf of all authors: all authors. Administrative/ technical/material support: all authors. Study supervision: Jovin.

\section{Disclosure Statement}

Dr. Jovin received grant, nonfinancial, and other support: Fundació Ictus Malaltia Vascular; Covidien/ Medtronic (nonfinancial support); Silk Road Medical and Air Liquide (personal fees); Covidien/Medtronic and Stryker Neurovascular (nonfinancial support) outside the submitted work.

This research received no specific grant from any funding agency in the public, commercial or not-forprofit sectors.

\section{References}

1 Higashida RT, Furlan AJ, Roberts H, Tomsick T, Connors B, Barr J, et al: Trial design and reporting standards for intra-arterial cerebral thrombolysis for acute ischemic stroke. Stroke 2003;34:e109-e137.

2 Berkhemer OA, Fransen PS, Beumer D, van den Berg LA, Lingsma HF, Yoo AJ, et al: A randomized trial of intraarterial treatment for acute ischemic stroke. N Engl J Med 2015;372:11-20.

3 Saver JL, Goyal M, Diener HC, Investigators SP: Stent-retriever thrombectomy for stroke. N Engl J Med 2015; 373:1077.

4 Broderick JP, Palesch YY, Demchuk AM, Yeatts SD, Khatri P, Hill MD, et al: Endovascular therapy after intravenous t-PA versus t-PA alone for stroke. N Engl J Med 2013;368:893-903.

5 Jankowitz B, Grandhi R, Horev A, Aghaebrahim A, Jadhav A, Linares G, et al: Primary manual aspiration thrombectomy (MAT) for acute ischemic stroke: safety, feasibility and outcomes in 112 consecutive patients. J Neurointerv Surg 2015;7:27-31.

6 Jankowitz B, Aghaebrahim A, Zirra A, Spataru O, Zaidi S, Jumaa M, et al: Manual aspiration thrombectomy: adjunctive endovascular recanalization technique in acute stroke interventions. Stroke 2012;43:1408-1411.

7 Turk AS, Spiotta A, Frei D, Mocco J, Baxter B, Fiorella D, et al: Initial clinical experience with the adapt technique: a direct aspiration first pass technique for stroke thrombectomy. J Neurointerv Surg 2014;6:231-237.

8 Mocco J, Khatri P, Zaidat 0: The randomized, concurrent controlled trial to assess the penumbra system's safety and effectiveness in the treatment of acute stroke. European Stroke Organisation (ESO) Conference, Glasgow, 2015.

9 Barber PA, Demchuk AM, Zhang J, Buchan AM: Validity and reliability of a quantitative computed tomography score in predicting outcome of hyperacute stroke before thrombolytic therapy. ASPECTS Study Group. Alberta Stroke Programme Early CT Score. Lancet 2000;355:1670-1674.

10 Berger C, Fiorelli M, Steiner T, Schabitz WR, Bozzao L, Bluhmki E, et al: Hemorrhagic transformation of ischemic brain tissue: asymptomatic or symptomatic? Stroke 2001;32:1330-1335.

11 Khatri P, Yeatts SD, Mazighi M, Broderick JP, Liebeskind DS, Demchuk AM, et al: Time to angiographic reperfusion and clinical outcome after acute ischaemic stroke: an analysis of data from the Interventional Management of Stroke (IMS III) phase 3 trial. Lancet Neurol 2014;13:567-574.

12 Sheth SA, Jahan R, Gralla J, Pereira VM, Nogueira RG, Levy EI, et al: Time to endovascular reperfusion and degree of disability in acute stroke. Ann Neurol 2015;78:584-593.

13 Patel RD, Saver JL: Evolution of reperfusion therapies for acute brain and acute myocardial ischemia: a systematic, comparative analysis. Stroke 2013;44:94-98. 
14 Nogueira RG, Lutsep HL, Gupta R, Jovin TG, Albers GW, Walker GA, et al: Trevo versus Merci retrievers for thrombectomy revascularisation of large vessel occlusions in acute ischaemic stroke (TREVO 2): a randomised trial. Lancet 2012;380:1231-1240.

15 Saver JL, Jahan R, Levy EI, Jovin TG, Baxter B, Nogueira RG, et al: Solitaire flow restoration device versus the merci retriever in patients with acute ischaemic stroke (SWIFT): a randomised, parallel-group, non-inferiority trial. Lancet 2012;380:1241-1249.

16 Pereira VM, Gralla J, Davalos A, Bonafe A, Castano C, Chapot R, et al: Prospective, multicenter, single-arm study of mechanical thrombectomy using solitaire flow restoration in acute ischemic stroke. Stroke 2013;44:28022807.

17 Zaidat 00, Castonguay AC, Gupta R, Sun CH, Martin C, Holloway WE, et al: North American Solitaire Stent Retriever Acute Stroke Registry: post-marketing revascularization and clinical outcome results. J Neurointerv Surg 2014;6:584-588.

18 Nguyen TN, Malisch T, Castonguay AC, Gupta R, Sun CH, Martin CO, et al: Balloon guide catheter improves revascularization and clinical outcomes with the solitaire device: analysis of the North American Solitaire Acute Stroke Registry. Stroke 2014;45:141-145.

19 Kirtane AJ, Stone GW: The Anchor-Tornus technique: a novel approach to 'uncrossable' chronic total occlusions. Catheter Cardiovasc Interv 2007;70:554-557.

20 Humphries W, Hoit D, Doss VT, Elijovich L, Frei D, Loy D, et al: Distal aspiration with retrievable stent assisted thrombectomy for the treatment of acute ischemic stroke. J Neurointerv Surg 2015;7:90-94. 